\title{
NUSTAR REVEALS RELATIVISTIC REFLECTION BUT NO ULTRA-FAST OUTFLOW IN THE QUASAR PG 1211+143
}

\author{
A. Zoghbi ${ }^{1}$, J. M. Miller ${ }^{1}$, D. J. WAlton $^{2,3}$, F. A. Harrison ${ }^{3}$, A. C. Fabian ${ }^{4}$, C. S. Reynolds ${ }^{5}$, S. E. Boggs ${ }^{6}$, \\ F. E. Christensen ${ }^{7}$, W. Craig $^{6}$, C. J. Hailey ${ }^{8}$, D. Stern $^{2}$, and W. W. Zhang ${ }^{9}$ \\ ${ }^{1}$ Department of Astronomy, University of Michigan, 1085 South University Avenue, Ann Arbor, MI 48109, USA; abzoghbi@umich.edu \\ ${ }^{2}$ Jet Propulsion Laboratory, California Institute of Technology, Pasadena, CA 91109, USA \\ ${ }^{3}$ Space Radiation Laboratory, California Institute of Technology, Pasadena, CA 91125, USA \\ ${ }^{4}$ Institute of Astronomy, University of Cambridge, Madingley Road, Cambridge, CB3 OHA, UK \\ ${ }^{5}$ Department of Astronomy, University of Maryland, College Park, MD 20742-2421, USA \\ ${ }^{6}$ Space Science Laboratory, University of California, Berkeley, CA 94720, USA \\ ${ }^{7}$ DTU Space. National Space Institute, Technical University of Denmark, Elektrovej 327, DK-2800 Lyngby, Denmark \\ ${ }^{8}$ Columbia Astrophysics Laboratory, Columbia University, New York, NY 10027, USA \\ ${ }^{9}$ NASA Goddard Space Flight Center, Greenbelt, MD 20771, USA \\ Received 2014 December 22; accepted 2015 January 6; published 2015 January 29
}

\begin{abstract}
We report on four epochs of observations of the quasar PG 1211+143 using NuSTAR. The net exposure time is 300 ks. Prior work on this source found suggestive evidence of an ultra-fast outflow (UFO) in the Fe K band with a velocity of approximately $0.1 c$. The putative flow would carry away a high-mass flux and kinetic power, with broad implications for feedback and black hole-galaxy co-evolution. NuSTAR detects PG 1211+143 out to $30 \mathrm{keV}$, meaning that the continuum is well-defined both through and above the Fe $\mathrm{K}$ band. A characteristic relativistic disk reflection spectrum is clearly revealed via a broad $\mathrm{Fe} \mathrm{K}$ emission line and Compton back-scattering curvature. The data offer only weak constraints on the spin of the black hole. A careful search for UFOs shows no significant absorption feature above $90 \%$ confidence. The limits are particularly tight when relativistic reflection is included. We discuss the statistics and the implications of these results in terms of connections between accretion onto quasars, Seyferts, and stellar-mass black holes, and feedback into their host environments.
\end{abstract}

Key words: accretion, accretion disks - galaxies: active - galaxies: nuclei - quasars: absorption lines quasars: individual (PG 1211+143)

\section{INTRODUCTION}

The observation of a relation between the masses of supermassive black holes at the centers of galaxies and the stellar velocity dispersion $(M-\sigma$ relation; Ferrarese \& Merritt 2000; Gultekin et al. 2009) suggests a direct link between black holes and their host galaxies. Energy and momentum driven out from the central regions push gas and dust away, halting star formation and stopping active galactic nucleus (AGN) fueling (Silk \& Rees 1998; Di Matteo et al. 2005; Churazov et al. 2005 see Fabian 2012 for a review). The action of AGN feedback could be achieved through the powerful radio jets in the kinetic mode (e.g., McNamara \& Nulsen 2007). In the radiative mode, accretion disks drive powerful winds that could contribute significantly to the energy budget of the black hole-galaxy system.

Observing the properties of such a wind is of great importance, particularly in X-rays where most of the radiation from the expelled material is produced. Although warm absorber winds are common in the X-ray spectra of AGNs (Reynolds 1997; Crenshaw et al. 2003; Blustin et al. 2005) with outflow velocities of $\sim 1000 \mathrm{~km} \mathrm{~s}^{-1}$ and column densities of $\log \left(N_{h}\right) \sim 20-23 \mathrm{~cm}^{-2}$, they are weak, providing only $\sim 0.01 \%$ of the AGN bolometric luminosity (Blustin et al. 2005). The more powerful winds seen in several objects with outflow velocities of $\sim 0.1 c$ and column densities of $\log \left(N_{h}\right)=24 \mathrm{~cm}^{-2}$ could carry power that is a few percent of the bolometric luminosity (Pounds et al. 2003; Reeves et al. 2003; Cappi et al. 2009; Chartas et al. 2009; Pounds \& Reeves 2009; Tombesi et al. 2010, 2012; Gofford et al. 2013).
These ultra-fast outflows (UFO) seem to be present in at least 35\% of observed AGNs in X-rays (Tombesi et al. 2010) However, this number could be an overestimate when alternative modeling and more conservative statistical analyses are considered (Kaspi \& Behar 2006; Vaughan \& Uttley 2008). Establishing how common these outflows are as well as their physical and geometrical properties is therefore crucial for understanding their contribution to the energy and momentum budget of black holes and their hosts. In this Letter, we present analysis of the NuSTAR (Harrison et al. 2013) observation of the quasar PG $1211+143$. NuSTAR band (3-79 keV) with the unprecedented sensitivity at hard $(>10 \mathrm{keV}) \mathrm{X}$-rays fixes the continuum and thus allows a meaningful search for blueshifted absorption below $10 \mathrm{keV}$.

PG $1211+143(z=0.0809)$ is the archetypical case for the UFOs in active galaxies. The first observation with $X M M$ Newton in 2001 showed evidence for highly blueshifted absorption lines that are reminiscent of mildly relativistic disk winds $(\sim 0.1 c$; Pounds et al. 2003). The same data set was analyzed by Kaspi \& Behar (2006), who find a best-fit outflow velocity of $3000 \mathrm{~km} \mathrm{~s}^{-1}$ instead of the high $24,000 \mathrm{~km} \mathrm{~s}^{-1}$. A Chandra LETG observation showed two redshifted (instead of blueshifted!) absorption lines at 4.56 and $5.33 \mathrm{keV}$ in the source frame (Reeves et al. 2005), which, when identified as the $\mathrm{H}$-like $\mathrm{K} \alpha$ lines, corresponds to inflowing velocities of $(0.2-0.4) c$. Later XMM-Newton observations in 2004 and 2007 showed weaker lines but seem to be consistent with the original observations (Pounds \& Reeves 2009), or possibly with no absorption lines at all (Tombesi et al. 2010). 

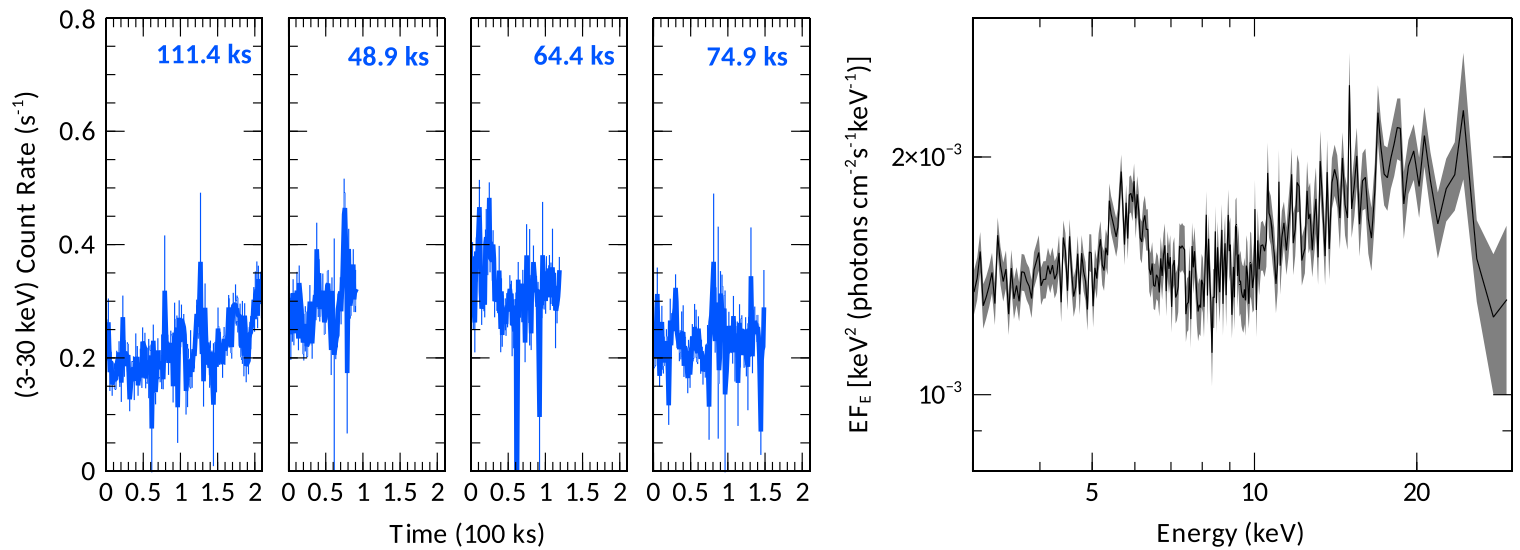

Figure 1. Left: 3-30 keV light curves from all the four NuSTAR observations of PG 1211+143. The net exposure is shown in each panel. Right: the spectrum from the combined observations (combining FPMA and FPMB from all observations) unfolded to a constant to remove the effective area of the detector, and plotted as EF $(E)$ using plot eeufspec in XSPEC.

\section{OBSERVATIONS AND DATA REDUCTION}

NuSTAR observed PG 1211+143 in four exposures between 2014 February and July (the exact dates are 2014 February 18, 2014 April 08 and 09, and 2014 July 07). The four observations had net exposures of $111,48,64$, and $74 \mathrm{ks}$, totaling to nearly $300 \mathrm{ks}$. The data were reduced using HEASOFT v6.16 with the latest calibration (version 20141020). We used the scripts nupipeline and nuproducts to extract the spectral products. Source and background spectra were extracted from regions on- (with a radius of 2 arcmin) and off-source, respectively, and grouped so that there are least 100 source counts per bin. The spectra were analyzed using XSPEC v12.8.2. Spectral analysis was performed on individual and combined spectra as discussed in Section 3. Spectra from the two focal point modules A and B (FPMA and FPMB) and from different epochs were combined using addspec tool in HEASOFT. The response files were combined using addrmf with the proper weighting.

The resulting 3-30 keV light curves from the four exposures are shown in Figure 1 (left). The average 3-10 flux (from a power-law fit) is $2.7 \times 10^{-12} \mathrm{ergs} \mathrm{cm}^{-2} \mathrm{~s}^{-1}$, which is about the same as the first XMM-Newton observation of 2001, which had a 3-10 keV flux of $2.5 \times 10^{-12} \mathrm{ergs} \mathrm{cm}^{-2} \mathrm{~s}^{-1}$. The source showed some flux variability between observations. No strong spectral changes are seen apart from a normalization change in the main power-law continuum (see Section 3).

\section{SPECTRAL ANALYSIS}

One of the goals of the NUSTAR observation was to search for absorption lines from high-velocity outflows. The spectrum from the new data sets is shown in Figure 1 (right). It has a clear iron $\mathrm{K}$ emission line and an excess above $10 \mathrm{keV}$ that is most likely due to the Compton reflection hump. To be systematic in the search, we consider several baseline models, including simple fits to the $3-10 \mathrm{keV}$ band, so we can directly compare with the baseline model used in Tombesi et al. (2010).

\subsection{Searching for Emission and Absorption Features}

In the following discussions, we search for absorption (and emission) features by adding a narrow ${ }^{10}$ Gaussian line and performing a systematic scan of the residuals for each baseline model. We use a grid of energies between 3.5 and $9.5 \mathrm{keV}$ in $100 \mathrm{eV}$ steps, and normalization values between $-1 \times 10^{-5}$ and $1 \times 10^{5}$ (to account for both emission and absorption). We use a Monte Carlo method to obtain significance estimates. As pointed out in Protassov et al. (2002), a simple $F$-test done by comparing the improvement in $\chi^{2}$ after adding a Gaussian line is not appropriate for obtaining meaningful statistical confidence intervals. The baseline model is not known a priori, and neither is the reference (null) distribution of the $F$-statistic.

This inapplicability of the $F$-test is not related to the quality of data, but rather it is fundamental to any case of an added spectral component. Using Monte Carlo methods provides a way of estimating the unknown reference distribution and then using it to assess the significance of deviations from the null model. The following steps are used to obtain a reference distribution that takes into account both the uncertainty in the baseline model parameters. These are similar to those in Porquet et al. (2004) and Tombesi et al. (2010) but additionally take into account the baseline model uncertainties.

A. Start with a baseline model with its best-fit $\chi_{0}^{2}$ and covariance matrix that encodes the uncertainty of best value parameters.

B. Draw $N$ random sets of parameters using the covariance matrix $(N=1000$ in our case). This can be achieved for instance by running a Markov Chain Monte Carlo parameter search and taking parameters directly from the resulting chains (after they have converged).

C. For each parameter set, fake spectra using the observed response and background files. This produces $N$ simulated spectra that are drawn from the baseline model taking into account its uncertainty.

D. Add a narrow Gaussian line and scan in energy and normalization and record the maximum improvement in $\Delta \chi^{2}$. This gives $N$ values of $\Delta \chi^{2}$ that are used to construct a reference distribution to which observed $\Delta \chi^{2}$ values are

\footnotetext{
${ }^{10}$ If a broad component is present in the residuals, several neighboring narrow lines will provide a $\chi^{2}$ improvements.
} 
Table 1

Fit Parameters for the Baseline Models Discussed in Section 3.1

\begin{tabular}{ll}
\hline \hline & powerlaw+zgauss (3-10 keV) \\
$E_{\text {zgauss }}$ & $6.28 \pm 0.07 \mathrm{keV}$ \\
$\sigma_{\text {zgauss }}$ & $0.33 \pm 0.07 \mathrm{keV}$ \\
\hline & powerlaw+reflionx (3-50 keV) \\
$A_{\mathrm{Fe}}($ solar $)$ & $0.7 \pm 0.1$ \\
$\Gamma$ & $2.45 \pm 0.06$ \\
$\log (\xi)$ & $1.9 \pm 0.2$ \\
\hline & powerlaw+xillver $(3-50 \mathrm{keV})$ \\
$A_{\mathrm{Fe}}($ solar $)$ & $0.5 \pm 0.1$ \\
$\Gamma$ & $2.50 \pm 0.06$ \\
$\log (\xi)$ & $1.3 \pm 0.4$ \\
\hline & \\
$q$ & relxill (3-50 keV) \\
$a$ & $2.2 \pm .5$ \\
Incl. & $-0.13 \pm 0.8$ \\
$\Gamma$ & $28 \pm 7$ \\
$\log (\xi)$ & $2.51 \pm 0.2$ \\
$A_{\mathrm{Fe}}($ solar $)$ & $1.3 \pm 0.9$ \\
$E_{\text {cut }}$ & $0.7 \pm 0.1$ \\
$\operatorname{Ref.~fract.~}$ & $124(\mathrm{keV})($ lower limit $)$ \\
\hline
\end{tabular}

compared. The significance of an observed $\Delta \chi_{\mathrm{obs}}^{2}$ is $1-N_{\left(\Delta \chi^{2}>\Delta \chi_{\text {obs }}^{2}\right)} / N$, where $N_{\left(\Delta \chi^{2}>\Delta \chi_{\text {obs }}^{2}\right)}$ is the number of simulated $\Delta \chi^{2}$ values greater than $\Delta \chi_{\mathrm{obs}}^{2}$.

Several baseline models are used to assess their effect on estimating absorption features.

1. powerlaw + zgauss. This consists of a power law plus a broad Gaussian fitted to the $3-10 \mathrm{keV}$ spectrum similar to those used in Tombesi et al. (2010) and Gofford et al. (2013).

2. powerlawtreflionx (Ross \& Fabian 2005).

3. powerlawtxillver (Garcia et al. 2014).

4. relxill (Dauser et al. 2013).

For models in $2-4$, the whole NUSTAR band where the source is significantly detected above background is used. In all models, Galactic absorption is fixed at $2.7 \times 10^{20} \mathrm{~cm}^{-2}$. For the relativistic reflection models, we assume a power-law emissivity and we fit for its index $(q)$ along with the black hole spin, photon index, ionization parameter, iron abundance, inclination, high-energy cutoff, and reflection fraction. The details of the model parameters are shown in Table 1. For each baseline model, we repeat steps A-D. The results are shown in Figure 2.

\subsection{Line Search Results}

It is clear from Figure 2 that there is no significant absorption feature that is persistent with all baseline models. The phenomenological description of the first model gives an excellent description to the data in the 3-10 keV band with no significant residuals. In the reflionx fit, an absorption feature at $7.1 \mathrm{keV}$ is apparent along with the emission feature at $5.8 \mathrm{keV}$. The same feature at $5.8 \mathrm{keV}$ persists for the xillver fit. Both the reflionx and xillver models account for the $\mathrm{Fe} \mathrm{K} \alpha$ emission at $6.4 \mathrm{keV}$. The residuals at $\sim 5.8$ indicate that additional emission is required redward of the line, suggesting line broadening. If broadening is included in the fit (relxill, relxill+xillver), the feature disappears.

The absorption in the reflionx fit at $7.1 \mathrm{keV}$ disappears as well when relativistic blurring is included. The last model (relxill+xillver) is included for completeness. It models reflection from both the inner (with relativistic broadening) and outer regions. Although the additional component improves the $\chi^{2}$ slightly compared to relxill alone, its requirement is not very significant. The conclusion from Figure 2 is that absorption features depend on the chosen continuum, and when the continuum is pinned down with NUSTAR coverage at high energies and the inclusion of relativistic reflection, no significant absorption is seen.

The analysis summarized in Figure 2 was done using a combination of all the spectra (FPMA and FPMB from four observations) to obtain the best signal. We tested for any possible flux dependency by grouping observations 1 and 4 together and 2 and 3 together (see light curve in Figure 1 (left)), and the results do not change; no additional absorption lines are required by the data.

\subsection{Comparison with XMM-Newton}

The first XMM-Newton observation in 2001 showed an absorption line at $\sim 7.1 \mathrm{keV}$ in the observer's frame plus possible additional lines at lower energies (Pounds et al. 2003). We reanalyzed this XMM-Newton data set using the same method discussed in Section 3.1 and find the line at $\sim 7.5 \mathrm{keV}$ to be significant at the $\sim 99 \%$ level with an equivalent width of $104 \pm 52 \mathrm{eV}$. The flux levels of the first XMM-Newton observation and the NUSTAR observation discussed here are comparable; therefore, we also assess whether an absorption line similar to that seen in XMM-Newton would have been detectable in the current NuSTAR data. We fitted the XMMNewton PN spectrum with a model consisting of a power law, relativistic reflection, and an absorption line. We then used this model to fake NUSTAR data, and followed the procedure of Section 3.1 to search for the absorption line. The line is detected significantly in this simulated data, showing that an absorption line would have been easily ( $>8 \sigma$ significance) detected if it was present in the data. Adding a Gaussian line to our best-fit model (relxill) with the energy and the width of absorption line seen in the XMM-Newton spectrum provides a fit improvement of $\Delta \chi^{2}=3$ for one degree of freedom. The equivalent width of this line is $22 \pm 22 \mathrm{eV}$.

Subsequent to the first observation, XMM-Newton observed PG 1211+143 several times, and when we analyze these observations self-consistently, none of them show any significant absorption features when using a baseline model consisting of a power law and a relativistic line.

\subsection{Relativistic Reflection Parameters}

The best-fit model to the NUSTAR data, shown in Figure 3, consists of an absorbed power law and relativistic reflection, both modeled with relxill. Only data below $10 \mathrm{keV}$ are shown to highlight the differences among models. As was clear from Figure 2, the data show an excess at $\sim 5 \mathrm{keV}$ when fitted with simple reflection models. The addition of relativistic blurring (relxill) accounts for the excess. The data, however, do not allow very strong constraints on the spin of the black hole. The parameters of the best-fit model are shown in Table 1. 


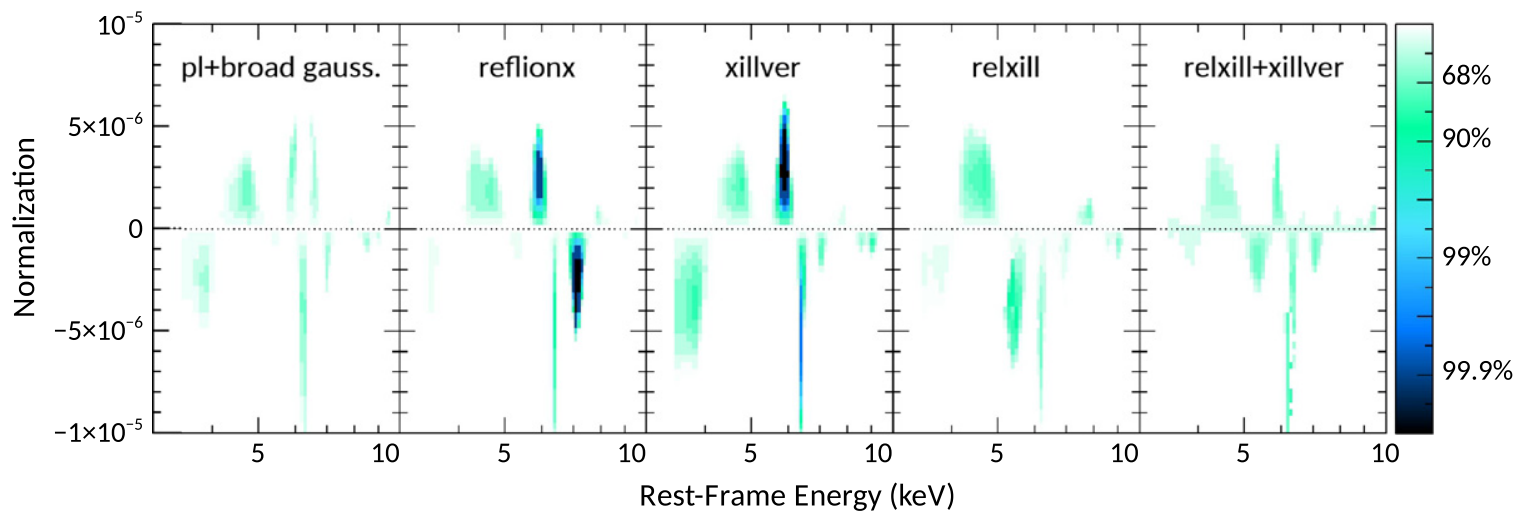

Figure 2. Line search significance plot for five baseline models. The regions with the darkest colors indicate that the null model (baseline without an additional emission or absorption feature) can be rejected with that significance. The color scale corresponds to $\log (1-$ significance $)$ and the corresponding significance values are shown in the color bar. The energy axis corresponds to the source reset frame energy $(z=0.0809)$.
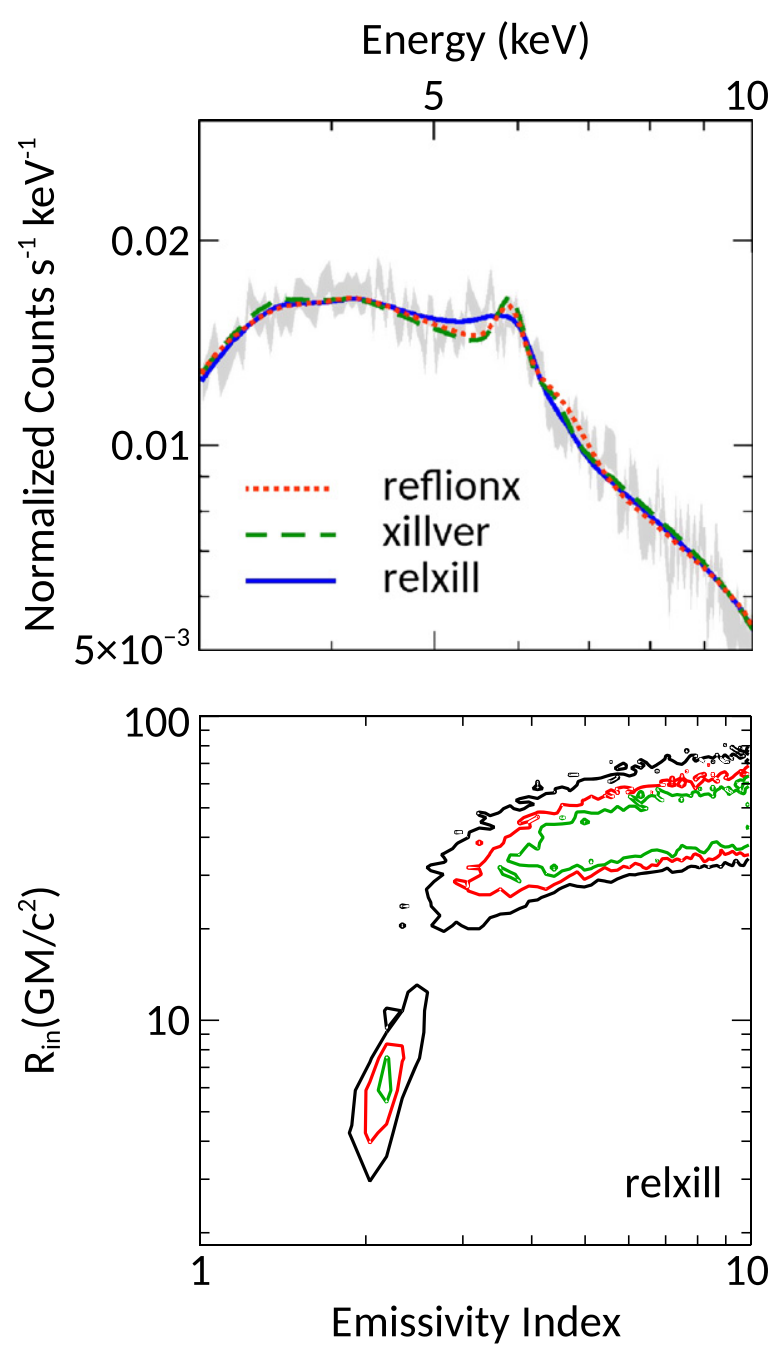

Figure 3. Top: the spectrum of PG 1211+143 along with the best-fit baseline models discussed in Section 3.1 plotted in the $3-10 \mathrm{keV}$ only where the models differ. The $x$-axis represents the rest-frame energy of the source. Bottom: marginal probability density contours in the relxill model for the inner radius of emission and the emissivity index. The contours are for the $68 \%$, $90 \%$, and $99 \%$ confidence levels.
If we fit for the inner radius of emission instead of the black hole spin, we obtain the marginal probability contours shown in the bottom panel of Figure 3. This figure is obtained by running a Monte Carlo Markov Chain ${ }^{11}$ on the best-fit relxill to obtain posterior probability distributions of the fit parameters and then marginalize over the parameters that are not of interest. The inner radius of emission and emissivity index are degenerate in the fit, but it is clear that although the extra broadening of the $\mathrm{Fe} \mathrm{K} \alpha$ line is required, it is not very large. The inner radius of emission ranges from $\sim 4$ to 40 gravitational radii $\left(r_{g}=G M / c^{2}\right)$ depending on the disk emissivity. The solution with a steep emissivity and large radii might be unphysical, and although this data do not allow us to firmly locate the X-ray emission, the detection of reverberation time delays in this objects in the XMM-Newton data (de Marco et al. 2011) would favor the solution with a small radius for the emission.

\section{DISCUSSION AND CONCLUSIONS}

We have analyzed a deep $300 \mathrm{ks}$ observation of the quasar PG 1211+143, obtained with NuSTAR in four time intervals spanning six months. When fit with a simple power-law model, the total spectrum shows clear signatures of disk reflection (Figure 1 (right)). Fits with the newest, most sophisticated reflection models currently available provide significantly improved fits. The residuals to fits with simple reflection show an excess around $\sim 5 \mathrm{keV}$, indicating that relativistic blurring is required, clearly signaling that $\mathrm{X}$-ray emission probes the very innermost portion of the accretion flow in PG 1211+143. The data do not permit a strong constraint on the spin of the black hole, but when reverberation delays seen previously in XMMNewton data are considered, a solution with a small inner radius ( $\sim 4 r_{g}$, corresponding to a black hole spin of $\sim 0.5$ ) is supported by the data. We thoroughly examined the NuSTAR data both in segments and in total, and the spectra show no compelling evidence of a steady or variable UFO seen previously in this source. The limits are particularly tight once disk reflection has been modeled.

NuSTAR clearly has the sensitivity and spectral resolution required to detect extraordinary outflows, when they are present. Observations of PDS 456, for instance, reveal a likely

\footnotetext{
$\overline{11}$ We use xspec_emcee available from https://github.com/jeremysanders/ xspec_emcee.
} 
P-Cygni profile in that quasar, signaling a massive and powerful outflow from that black hole into its host galaxy (Nardini et al. 2014). NuSTAR spectra from observations of NGC 1365 were able to disentangle variable absorption, outflows, and disk reflection in that source, leading to a robust measure of the black hole spin (Risaliti et al. 2013; Walton et al. 2014). At the opposite end of the mass scale, NuSTAR observations of the black hole candidate 4U 1630-472 revealed strong absorption that may be a UFO-like outflow, in addition to disk reflection (King et al. 2014). The true extent of these UFOs and their persistence is unclear.

The non-detection of UFOs in the prototypical object PG $1211+143$ in the current observation (and also previous observations subsequent to the first XMM-Newton detection) raises several questions on how common these might be in this and other sources. Systematic searches suggest that $\sim 40 \%$ of objects observed with XMM-Newton and Suzaku show them (Tombesi et al. 2010; Gofford et al. 2013). This number could be much lower if a careful statistical search is performed that takes into account physical baseline models along with their observational uncertainties. This comes in the form of including the best-fit uncertainties in simulating spectra during the Monte Carlo tests and also allowing for that uncertainty when fitting for narrow features at arbitrary energies in the spectrum.

The true extent of UFOs could be affected by the significance of any single detection, which we have discussed, and also by their variability. A non-detection could simply be due to the fact they are transient in nature as suggested by the the simulations (Proga \& Kallman 2004). The case of PG 1211 +143 shows that the UFO is seen in 1 out of 11 observations (XMM-Newton, Suzaku, Chandra, and NuSTAR) between 2001 and 2014. With the caveat that we still have small numbers, if one assumes the observations are done randomly as far as the AGN is concerned, it appears that a variability argument would also suggest that the contribution of UFO's is an order of magnitude lower than previously inferred.
This work made use of data from the NUSTAR mission, a project led by the California Institute of Technology, managed by the Jet Propulsion Laboratory, and funded by the National Aeronautics and Space Administration.

\section{REFERENCES}

Blustin, A. J., Page, M. J., Fuerst, S. V., Branduardi-Raymont, G., \& Ashton, C. E. 2005, A\&A, 431, 111

Cappi, M., Tombesi, F., Bianchi, S., et al. 2009, A\&A, 504, 401

Chartas, G., Saez, C., Brandt, W. N., Giustini, M., \& Garmire, G. P. 2009, ApJ, 706, 644

Churazov, E., Sazonov, S., Sunyaev, R., et al. 2005, MNRAS, 363, L91

Crenshaw, D. M., Kraemer, S. B., \& George, I. M. 2003, ARA\&A, 41, 117

Dauser, T., Garcia, J., Wilms, J., et al. 2013, MNRAS, 430, 1694

de Marco, B., Ponti, G., Uttley, P., et al. 2011, MNRAS, 417, L98

Di Matteo, T., Springel, V., \& Hernquist, L. 2005, Natur, 433, 604

Fabian, A. C. 2012, ARA\&A, 50, 455

Ferrarese, L., \& Merritt, D. 2000, ApJL, 539, L9

García, J., Dauser, T., Lohfink, A., et al. 2014, ApJ, 782, 76

Gofford, J., Reeves, J. N., Tombesi, F., et al. 2013, MNRAS, 430, 60

Gültekin, K., Richstone, D. O., Gebhardt, K., et al. 2009, ApJ, 698, 198

Harrison, F. A., Craig, W. W., Christensen, F. E., et al. 2013, ApJ, 770, 103

Kaspi, S., \& Behar, E. 2006, ApJ, 636, 674

King, A. L., Walton, D. J., Miller, J. M., et al. 2014, ApJL, 784, L2

McNamara, B. R., \& Nulsen, P. E. J. 2007, ARA\&A, 45, 117

Nardini, E., et al. 2014, Natur, in press

Porquet, D., Reeves, J. N., Uttley, P., \& Turner, T. J. 2004, A\&A, 427, 101

Pounds, K. A., \& Reeves, J. N. 2009, MNRAS, 397, 249

Pounds, K. A., Reeves, J. N., King, A. R., et al. 2003, MNRAS, 345, 705

Proga, D., \& Kallman, T. R. 2004, ApJ, 616, 688

Protassov, R., van Dyk, D. A., Connors, A., Kashyap, V. L., \& Siemiginowska, A. 2002, ApJ, 571, 545

Reeves, J. N., O'Brien, P. T., \& Ward, M. J. 2003, ApJL, 593, L65

Reeves, J. N., Pounds, K., Uttley, P., et al. 2005, ApJL, 633, L81

Reynolds, C. S. 1997, MNRAS, 286, 513

Risaliti, G., Harrison, F. A., Madsen, K. K., et al. 2013, Natur, 494, 449

Ross, R. R., \& Fabian, A. C. 2005, MNRAS, 358, 211

Silk, J., \& Rees, M. J. 1998, A\&A, 331, L1

Tombesi, F., Cappi, M., Reeves, J. N., \& Braito, V. 2012, MNRAS, 422, L1

Tombesi, F., Cappi, M., Reeves, J. N., et al. 2010, A\&A, 521, A57

Vaughan, S., \& Uttley, P. 2008, MNRAS, 390, 421

Walton, D. J., Risaliti, G., Harrison, F. A., et al. 2014, ApJ, 788, 76 